\title{
Abnormal Cerebral Microstructure in Premature Neonates with Congenital Heart Disease
}

\author{
L.B. Paquette, J.L. Wisnowski, R. Ceschin, J.D. Pruetz, J.A. Detterich, S. Del Castillo, A.C. Nagasunder, R. Kim, M.J. Painter, F.H. Gilles,
} M.D. Nelson, R.G. Williams, S. Blüml, and A. Panigrahy

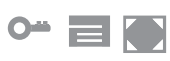

\begin{abstract}
BACKGROUND AND PURPOSE: Abnormal cerebral microstructure has been documented in term neonates with congenital heart disease, portending risk for injury and poor neurodevelopmental outcome. Our hypothesis was that preterm neonates with congenital heart disease would demonstrate diffuse cerebral microstructural abnormalities when compared with critically ill neonates without congenital heart disease. A secondary aim was to identify any association between microstructural abnormalities, white matter injury (eg, punctate white matter lesions), and other clinical variables, including heart lesions.
\end{abstract}

MATERIALS AND METHODS: With the use of tract-based spatial statistics, an unbiased, voxelwise method for analyzing diffusion tensor imaging data, we compared 21 preterm neonates with congenital heart disease with 2 cohorts of neonates without congenital heart disease: 28 term and 27 preterm neonates, identified from the same neonatal intensive care unit.

RESULTS: Compared with term neonates without congenital heart disease, preterm neonates with congenital heart disease had microstructural abnormalities in widespread regions of the central white matter. However, $42 \%$ of the preterm neonates with congenital heart disease had punctate white matter lesions. When neonates with punctate white matter lesions were excluded, microstructural abnormalities remained only in the splenium. Preterm neonates with congenital heart disease had similar microstructure to preterm neonates without congenital heart disease.

CONCLUSIONS: Diffuse microstructural abnormalities were observed in preterm neonates with congenital heart disease, strongly associated with punctate white matter lesions. Independently, regional vulnerability of the splenium, a structure associated with visual spatial function, was observed in all preterm neonates with congenital heart disease.

ABBREVIATIONS: $\mathrm{CHD}=$ congenital heart disease; $\mathrm{pWML}=$ punctate white matter lesion; $\mathrm{TBSS}=$ tract-based spatial statistics; $\mathrm{FA}=$ fractional anisotropy

$\mathbf{A}^{\mathrm{s}}$ the overall survival rate has improved for infants with congenital heart disease (CHD), there have been increased efforts made to improve long-term morbidity in the context of coexisting risk factors. ${ }^{1}$ Approximately $16 \%$ of infants with $\mathrm{CHD}$ are

Received December 10, 2012; accepted after revision January 8, 2013.

From the Division of Neonatology (L.B.P.); Department of Radiology (J.L.W., A.C.N., M.D.N., S.B., A.P.); Division of Cardiology (J.D.P., J.A.D.), Department of Pediatrics; Department of Anesthesiology and Critical Care Medicine (S.D.C.); Division of Cardio-Thoracic Surgery (R.K., R.G.W.), Department of Surgery; Division of Neuropathology (S.D.C.); and Department of Pathology (F.H.G.), Children's Hospital, Los Angeles, California; Brain and Creativity Institute (J.L.W.) and Department of Biomedical Engineering (S.B.), University of Southern California, Los Angeles, California; Departments of Pediatric Radiology (R.C., A.P.) and Pediatrics (M.J.P.), Division of Neurology, Children's Hospital of Pittsburgh of UPMC, and Department of Biomedical Informatics (R.C.), University of Pittsburgh, Pittsburgh, Pennsylvania.

L.B. Paquette and J.L. Wisnowski are co-first authors.

Support for this study is provided by the National Institutes of Health K23NS063371 to A.P., P50NA019632 to J.L.W., IULIRR031986 to A.P., J.L.W., and L.B.P., K12HD052954 to J.A.D.; and Rudi Schulte Foundation to S.B.

Please address correspondence to Ashok Panigrahy, MD, Department of Pediatric Radiology, Children's Hospital of Pittsburgh of UPMC, $45^{\text {th }}$ St and Penn Ave, Pittsburgh, PA 15201; e-mail: panigrahya@upmc.edu born prematurely, and CHD is twice as common in preterm compared with term neonates. ${ }^{2}$ Multiple studies have documented higher rates of mortality and morbidity in neonates with $\mathrm{CHD}$ who are born prematurely or at lower birth weight, ${ }^{2-6}$ and it has been shown that lower gestational age at birth is associated with worse neurodevelopmental outcome among infants with $\mathrm{CHD} .^{7,8}$

Prematurity and CHD are independent risk factors for adverse neurodevelopmental outcome, and it follows that having both further increases the risk. Prior studies of term neonates with CHD have also documented delayed brain maturation, particularly in term infants with single ventricle physiology, further increasing their risk for injury or adverse neurodevelopmental outcome. $^{9-15}$ Thus, examining the cerebral microstructure of preterm infants with CHD in relation to prematurity and injury

\footnotetext{
- Indicates open access to non-subscribers at www.ajnr.org

$\equiv$ Indicates article with supplemental on-line appendix.

Indicates article with supplemental on-line figures.

http://dx.doi.org/10.3174/ajnr.A3528
} 
may provide important insights into the complex combination of risks portending adverse neurodevelopment in infants with $\mathrm{CHD}$. To date, few neonatal neuroimaging studies have been described in preterm infants with CHD.

On neuropathologic examination, periventricular leukomalacia is a commonly observed finding in neonates with CHD and neonates with a history of preterm birth. ${ }^{16-21}$ With advances in neonatal intensive care, there has been a trend away from observations of cavitated lesions toward smaller noncavitated lesions (often described as "microcysts"). ${ }^{22}$ The most widely recognized MR correlate of noncavitated periventricular leukomalacia are punctate T1-hyperintense lesions. ${ }^{23}$

In the present study, we used DTI to investigate the microstructural integrity of the cerebral white matter and select gray matter regions in preterm neonates with CHD. Our hypothesis was that preterm neonates with $\mathrm{CHD}$ would demonstrate diffuse cerebral microstructural abnormalities at near term-equivalent age when compared with other critically ill neonates without CHD. Our secondary aim was to identify any association between microstructural abnormalities, documented patterns of white matter injury, and other pertinent clinical variables, including the complexity of the heart lesion. We included 2 separate comparison groups: term neonates without $\mathrm{CHD}$ and preterm neonates without CHD, both of which were identified from the same highrisk neonatal intensive care unit. The term neonates without $\mathrm{CHD}$ allowed us to determine whether the microstructure in our preterm neonates with CHD differed from "normal" at term-equivalent age. The preterm neonates without CHD allowed us to determine whether the presence of a congenital heart defect resulted in further microstructural abnormality than would be accounted for by prematurity alone. Finally, by separating our preterm neonates with CHD into the subset with punctate white matter lesions (pWMLs) and those without pWMLs, we were able to directly address whether the microstructural abnormalities were related to cerebral white matter injury.

\section{MATERIALS AND METHODS Subjects}

Target Cohort of Preterm Neonates with CHD. Neonates undergoing clinically indicated brain MRI at near term-equivalent age during the period of 2005-2010 were recruited as part of ongoing longitudinal studies of neurodevelopment in neonates with prematurity and $\mathrm{CHD}$ at a single institution. In the preterm $\mathrm{CHD}$ group, we included neonates with any heart anomaly treated surgically, including atrial septal defect, ventricular septal defect, patent ductus arteriosus requiring surgery after term equivalency, hypoplastic left heart syndrome, Ebstein anomaly, coarctation of the aorta, truncus arteriosus, transposition of the great arteries, and double-outlet right ventricle (Table 1). Patients with CHD were excluded if 1 ) the heart anomaly did not require surgery; 2 ) they had a chromosomal abnormality; 3 ) the brain MR imaging did not include DTI data that were analyzable (ie, caused by motion artifact or technical factors); and 4) there was a congenital brain malformation or a significant brain abnormality/injury, which could distort subsequent DTI measurements. Given the diversity of heart lesions treated by surgery in the preterm CHD group, the clinical data (including fetal echocardiograms) for the
Table 1: Preterm CHD cases_frequency of diagnoses

\begin{tabular}{lc}
\multicolumn{1}{c}{ Heart Defects } & $\boldsymbol{n}$ \\
\hline Hypoplastic left heart syndrome & 5 \\
Ebstein anomaly & 2 \\
Coarctation of the aorta & 3 \\
Transposition of the great arteries & 2 \\
Atrial septal defect, ventricular septal defect, patent ductus & 8 \\
$\quad$ arteriosus requiring surgery & 1 \\
Double-outlet right ventricle & \\
\hline
\end{tabular}

preterm CHD cases were reviewed by 3 pediatric cardiologists (J.D.P., R.G.W., J.A.D.) for determination of heart lesion complexity (see more details in On-line Appendix).

Comparison Cohorts of Critically Ill Term and Preterm Neonates without CHD. For comparison, we included data from 2 cohorts of neonates without CHD identified from the same high-risk neonatal intensive care unit-the first obtained as part of an internal review board-approved retrospective review of neonatal MRI conducted at the same institution as above between 2005-2010 and the second obtained as part of an ongoing longitudinal research program focused on prematurity. All near term-equivalent MRI was completed under clinical indications. The clinical indication for these studies included assessment of abnormal brain morphology (not confirmed), assessment of suspected brain injury or infection (not confirmed by imaging or relevant laboratory studies), assessment of possible seizure activity (including febrile seizures), and assessment of a nonintracranial abnormality, including a facial or orbital lesion. For all neonates, medical records were reviewed, including pertinent outpatient follow-up, by a neonatologist (L.B.P.) and a pediatric neuropsychologist (J.L.W.) (On-line Appendix). We also used region of interest measurements to validate this group against published healthy neonatal DTI data by using the same methods (On-line Appendix). ${ }^{24}$

\section{MR Imaging Protocol}

All imaging was obtained in a $1.5 \mathrm{~T}$ system (GE Healthcare Medical Systems, Milwaukee, Wisconsin) with a neonatal head coil and neonatal incubator (if clinically necessary). The following imaging sequences were acquired: T2WI FSE in axial and coronal planes $(\mathrm{TE} / \mathrm{TR}=85 / 5000 \mathrm{~ms}, \mathrm{FOV}=20 \mathrm{~cm}$, matrix $=320 \times 160$ or $256 \times 128$, section thickness, $3 \mathrm{~mm}$, spacing $=0)(5$ minutes $)$; coronal T1 3D spoiled gradient-recalled echo $(\mathrm{TE} / \mathrm{TR}=6 / 25 \mathrm{~ms}$, $\mathrm{FOV}=18 \mathrm{~cm}$, matrix $=256 \times 160$, section thickness $=1 \mathrm{~mm}$, spacing $=0)(10$ minutes $)$. The DTI protocol included an EPI sequence with the following parameters: TE/TR $=80 / 10,000 \mathrm{~ms}$, FOV $=22 \mathrm{~cm}$, matrix $=128 \times 128$, section thickness $=4.5 \mathrm{~mm}$, spacing $=0$, with an in-plane resolution of $1.7 \mathrm{~mm}$ applied along 25 noncolinear directions with a b-value of 700 seconds $/ \mathrm{mm}^{2}$ (6 minutes).

\section{Evaluation of Conventional MR Imaging Data and Identification of $p W M L s$}

All standard MR images were reviewed by 2 independent readers (J.L.W., A.P.) for the presence of pWMLs, used here as a MR imaging biomarker for noncystic periventricular leukomalacia (Fig 1). Consensus was achieved if there was disagreement.

AJNR Am J Neuroradiol 34:2026-33 Oct 2013 www.ajnr.org 


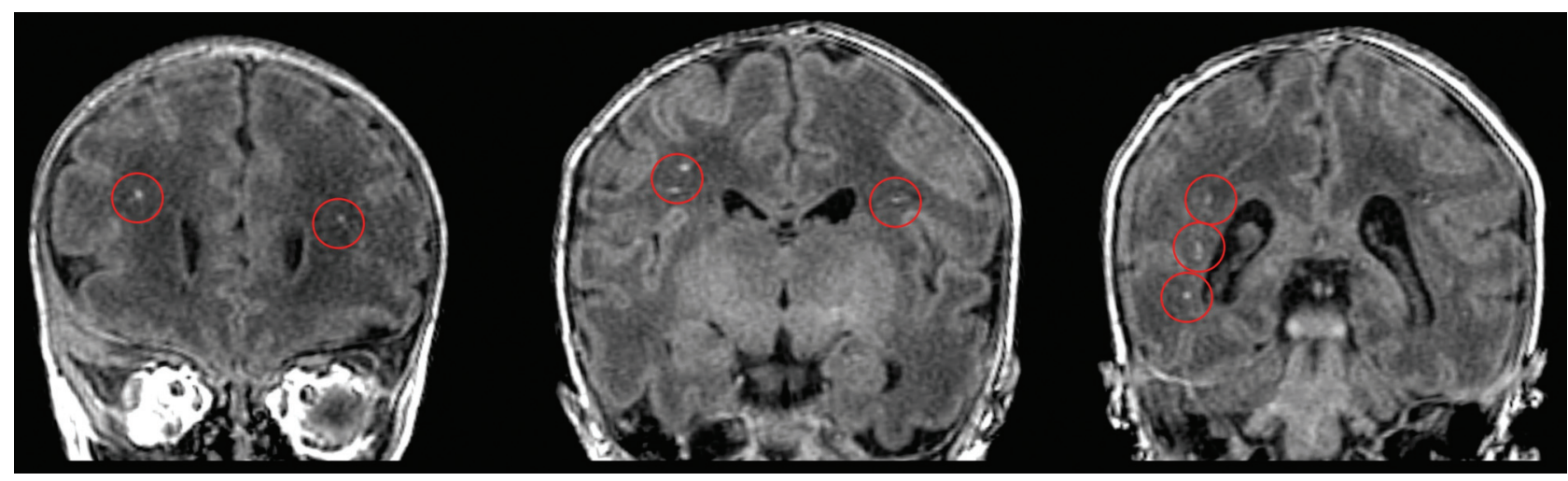

FIG 1. Infant with hypoplastic left heart syndrome born at 31 weeks postconceptional age and imaged at 35.5 weeks postconceptional age. Three coronal cuts from the 3D T7-weighted spoiled gradient recalled echo sequence demonstrating punctate Th-hyperintense lesions (circled in red) in the periventricular white matter and corona radiata just rostral to the genu of the corpus callosum (left), at the level of the pre- and post-central gyri (middle), and posterior to the trigone of the lateral ventricle (right), consistent with bilateral pWMLs or periventricular leukomalacia.

Table 2: Comparison of clinical variables between preterm neonates with CHD with PWMLs and preterm neonates without CHD

\begin{tabular}{|c|c|c|c|}
\hline & $\begin{array}{l}\text { CHD Preterm with } \\
\text { pWMLs }(n=8)\end{array}$ & $\begin{array}{l}\text { Control Preterm } \\
\qquad(n=27)\end{array}$ & $P$ Value $^{\mathrm{a}}$ \\
\hline PCA, weeks, mean (SD) & $38.63(3.36)$ & $42.61(6.51)$ & $.13^{\mathrm{a}}$ \\
\hline GA, weeks, mean (SD) & $33.29(1.89)$ & $30.67(4.62)$ & $.156^{\mathrm{a}}$ \\
\hline PNA MRI, weeks, mean (SD) & $5.35(4.38)$ & $11.095(8.24)$ & $.087^{\mathrm{a}}$ \\
\hline PCA MRI, weeks, mean (SD) & $38.63(3.36)$ & $42.61(6.51)$ & $.13^{\mathrm{a}}$ \\
\hline Apgar 1 minute, median $(n)$ & $6(6)$ & $6(21)$ & $.860^{\mathrm{c}}$ \\
\hline Apgar 5 minutes, median $(n)$ & $8.5(6)$ & $8(21)$ & $.878^{c}$ \\
\hline Apgar 10 minutes, median $(n)$ & $6(2)$ & $7(3)$ & $.747^{\mathrm{c}}$ \\
\hline Size for GA & 33 & 8 & $.216^{\mathrm{b}}$ \\
\hline Small, \% & 67 & 84 & \\
\hline Appropriate, \% & 0 & 8 & \\
\hline Large, \% & 6 & 25 & \\
\hline ISAM, \% (n) & $0(5)$ & $8.3(24)$ & $.763^{d}$ \\
\hline Postnatal sepsis, \% (n) & $42.9(7)$ & $60.0(25)$ & $.706^{\mathrm{d}}$ \\
\hline Hydrocortisone for BP, \% (n) & $50(6)$ & $18.5(27)$ & $.271^{\mathrm{d}}$ \\
\hline Days on hydrocortisone, mean (SD) & $0.5(0.55)$ & $1.741(6.06)$ & $.62^{\mathrm{a}}$ \\
\hline Inotropes, \% (n) & $100(6)$ & $46.2(26)$ & $.02^{\mathrm{a}}$ \\
\hline Days on dopamine, mean (SD) & $4.33(4.23)$ & $3.81(5.98)$ & $.84^{\mathrm{a}}$ \\
\hline
\end{tabular}

Note:-PCA indicates postconceptional age; GA, gestational age; PNA, postnatal age; ISAM, infant of substance abusing mom; BP, blood pressure.

${ }^{a}$ ANOVA $P$ values $\leq .005$

${ }^{\mathrm{b}}$ Measurements are average left and right

"Mann-Whitney for testing difference in medians.

${ }^{\mathrm{d}} \chi^{2}$ for tests of homogeneity

\section{DTI Postprocessing: Tract-Based Spatial Statistics}

DTI data analysis was performed by using FSL software (Version 4.1.4; http://www.fmrib.ox.ac.uk/fsl $)^{25}$. Our protocol for tractbased spatial statistics (TBSS) was similar to other published methods previously optimized for the neonates. ${ }^{26}$ (Please refer to On-line Appendix for more detail about TBSS methodology).

\section{Additional Statistical Analyses}

Continuous clinical variables were compared by use of a 3-way ANOVA. Pair-wise comparisons were then made by means of Tukey analysis. Categoric clinical variables were compared by means of Kruskal-Wallis or $\chi^{2}$ tests, as appropriate. Differences in perioperative variables were determined by nonparametric tests. R Developmental Core Team (http://www.r-project.org/) and SPSS (Version 19; SPSS, Chicago, Illinois) were used for all statistical analyses.

\section{RESULTS}

\section{Characterization of the Clinical Population and Clinical Variable Data}

There were 76 cases that met the inclusion criteria for this study: 21 preterm neonates with CHD, 28 neonates born at term ( $\geq 37$ weeks; term comparison group), and 27 neonates born between 23-36 weeks gestational age (preterm comparison group). There were no significant differences in postconceptional age (defined as gestational age at birth plus postnatal age) at time of MR imaging among the 3 groups, including the subset of preterm CHD cases identified with pWMLs $(P=.58$, ANOVA). Information regarding diagnosis, clinical course, MR imaging, and surgical variables for preterm CHD cases are summarized in Tables 1-3.

\section{Incidence of $p W M L s$ in the Preterm CHD Group}

On the basis of conventional MR imaging, pWMLs were found in approximately $42 \%$ of the preterm CHD cases $(\kappa$ score $=1.0)($ Fig $1)$. Of the CHD cases with pWMLs, $63 \%$ had MR imaging performed in the postoperative period. The most common heart lesion in the preterm neonates with CHD with pWMLs was hypoplastic left heart syndrome $(n=4 ; 1$ preoperative; 3 postoperative). The other diagnoses associated with pWMLs included 1) Ebstein anomaly ( $n=1$, preoperative); coarctation ( $n=1$, postoperative); ventricular septal defect $(n=1$, preoperative); and double-outlet right ventricle ( $n=1$, postoperative). The highest number of pWMLs was noted in 2 hypoplastic left heart syndrome cases and a coarctation case. We compared clinical variables between the preterm neonates with $\mathrm{CHD}$ and pWMLs and the preterm neonates without CHD, and the only statistically significant difference was the relatively increased use of inotropes $(P<.02)$ (Table 2$)$. We also compared multiple 
Table 3: Preterm CHD group: comparison of perioperative variables between $\mathrm{pWML}$ versus non-pWML groups

\begin{tabular}{|c|c|c|c|c|c|}
\hline \multirow[b]{2}{*}{ Surgical Variables } & \multicolumn{2}{|c|}{$\begin{array}{c}\text { CHD Preterm Group } \\
\text { with pWML }\end{array}$} & \multicolumn{2}{|c|}{$\begin{array}{c}\text { CHD Preterm } \\
\text { without } \mathrm{PWML}\end{array}$} & \multirow[b]{2}{*}{$P$ Value } \\
\hline & Mean (SD) & $n$ & Mean (SD) & $n$ & \\
\hline Birth weight & $1963.5(591.6)$ & 8 & $1676.2(566.6)$ & 11 & .341 \\
\hline Age at $1^{\text {st }}$ surgery, days & $3.5(2.8)$ & 8 & $12.0(12.7)$ & 12 & .05 \\
\hline PCA at $1^{\text {st }}$ surgery, weeks & $37(1.7)$ & 8 & 43.1 (10.9) & 12 & .20 \\
\hline $1^{\text {st }} \mathrm{ABG} \mathrm{pH}$ & $7.24(0.08)$ & 5 & $7.29(1.10)$ & 5 & .36 \\
\hline $1^{\mathrm{st}} \mathrm{ABG} \mathrm{pO}_{2}$ & $81.75(74.82)$ & 4 & $39(-)$ & 1 & .64 \\
\hline Pre-op ABG pH & $7.33(0.1)$ & 8 & $7.36(0.05)$ & 12 & .41 \\
\hline Pre-op $\mathrm{ABG} \mathrm{pO}_{2}$ & $62.2(21.2)$ & 8 & $69.75(34.6)$ & 8 & .67 \\
\hline Post-op ABG pH & $7.30(0.07)$ & 8 & $7.32(0.05)$ & 12 & .38 \\
\hline Post-op ABG $\mathrm{pO}_{2}$ & $67.83(48.3)$ & 8 & $101(83.52)$ & 12 & .37 \\
\hline Pre-op epi, no. of days & $0(0)$ & 8 & $0.08(0.3)$ & 12 & .49 \\
\hline Pre-op dobutamine, no. of days & $0(0)$ & 8 & $0.08(0.3)$ & 12 & .49 \\
\hline Pre-op dopamine, no. of days & $1.167(0.41)$ & 8 & $0.417(1.2)$ & 12 & .62 \\
\hline Pre-op hydrocortisone, no. of days & $0.167(0.41)$ & 8 & $0.08(0.3)$ & 12 & .61 \\
\hline Pre-op milirinone, no. of days & $0(0)$ & 8 & $0.08(0.3)$ & 12 & .49 \\
\hline Post-op epi, no. of days & $1.67(2.7)$ & 8 & $1.5(3)$ & 12 & .90 \\
\hline Post-op dobutamine, no. of days & $0(0)$ & 8 & $0.25(0.5)$ & 12 & .20 \\
\hline Post-op dopamine, no. of days & $4.17(4.4)$ & 8 & $3.17(4.3)$ & 12 & .64 \\
\hline Post-op hydrocortisone, no. of days & $0.33(0.5)$ & 8 & $1.67(5.5)$ & 12 & .50 \\
\hline Post-op milirinone, no. of days & $3.33(4.13)$ & 8 & $3.5(4.0)$ & 12 & .93 \\
\hline Post-op nitroprusside, no. of days & $0.0(0)$ & 8 & $0.33(0.9)$ & 12 & .37 \\
\hline
\end{tabular}

Note:-PCA indicates postconceptional age; ABG, arterial blood gas; epi, epinephrine; Pre-op, preoperative; Post-op, postoperative.

perioperative variables between the preterm CHD cases with pWMLs and the preterm CHD cases without pWMLs and found that $\mathrm{pWMLs}$ were associated with earlier age at surgery $(P<.05)$ (Table 3).

\section{TBSS: Preterm Neonates with CHD Versus Term Neonates without CHD}

Compared with the term neonates without $\mathrm{CHD}$, the preterm neonates with CHD were found to have significantly reduced fractional anisotrophy (FA) in focal regions of the posterior cerebral white matter. This included key regions caudally within the developing visual system (ie, optic radiations and the splenium of the corpus callosum) with relative sparing of the more rostral visual areas (ie, the inferior frontal-occipital fasciculus) (Fig 2, top row; and On-line Appendix Fig 2). There were fewer significantly reduced FA voxels seen in the developing limbic system (ie, fimbria and fornix) and the posterior body of the corpus callosum. There was relative sparing of the developing motor system (ie, posterior limb of the internal capsule and cerebral peduncle).

In contrast with the FA results, changes in diffusivity, including increases in both axial diffusivity and radial diffusivity, were observed in widespread cerebral white matter regions in the preterm neonates with CHD (Fig 2 and On-line Appendix Figs 3 and 4). There was involvement of all neural systems noted including both rostral and caudal visual areas, the limbic system, the motor system, and frontal lobe regions (Fig 2 and On-line Appendix Figs 3 and 4$)$.

To examine whether the differences in the DTI metrics of brain microstructure above reflected maturational differences, we excluded the preterm CHD cases with pWMLs and compared the remaining preterm CHD cases $(n=13)$ to the term neonates without CHD. In this analysis, most of the diffuse microstructural differences disappeared except for persistent decreased FA and increased radial diffusivity in the splenium (On-line Fig 2, bottom row).

\section{TBSS Analysis: Preterm Neonates with CHD Versus Preterm Neonates without CHD}

In contrast to the results for the comparison between the patients with CHD and the term comparison group, there were far fewer differences in the white matter of the preterm patients with $\mathrm{CHD}$ relative to preterm neonates without $\mathrm{CHD}$. In the analysis corrected for multiple comparisons, no significant differences were detected for FA or any of the diffusivity metrics.

To address whether there were maturation differences between the preterm neonates with CHD and the neonates without $\mathrm{CHD}$, we repeated the above analyses while excluding the preterm neonates with CHD with pWMLs. Notably, no significant differences were detected in any brain region for any DTI metric in either the corrected or uncorrected data.

TBSS Analysis: Complex CHD Preterm Cases Compared with Other Heart Lesions. We compared complex preterm CHD cases (hypoplastic left heart syndrome, transposition of the great arteries, and Ebstein with significant valvular disturbance) with the other preterm CHD anomalies (atrial septal defect, ventricular septal defect, patent ductus arteriosis, coarctation, double-outlet right ventricle with subaortic ventricular septal defect). There were diffuse microstructural abnormalities, including decreased FA and increased axial diffusivity and radial diffusivity in widespread white matter regions, in the single-ventricle physiology cases compared with the other CHD cases (Fig 3, top row). However, when the analysis was corrected for the number of pWMLs, no differences were detected between these 2 groups (Fig 3, bottom row). Of note, there was relative sparing of the corpus callosum, including the splenium, suggesting that splenium was microstructurally similar between the 2 preterm CHD groups (Fig 3). Similar results were also obtained when the hypoplastic left heart syndrome and transposition cases were grouped together and compared with all of the other heart lesions (data not shown). 


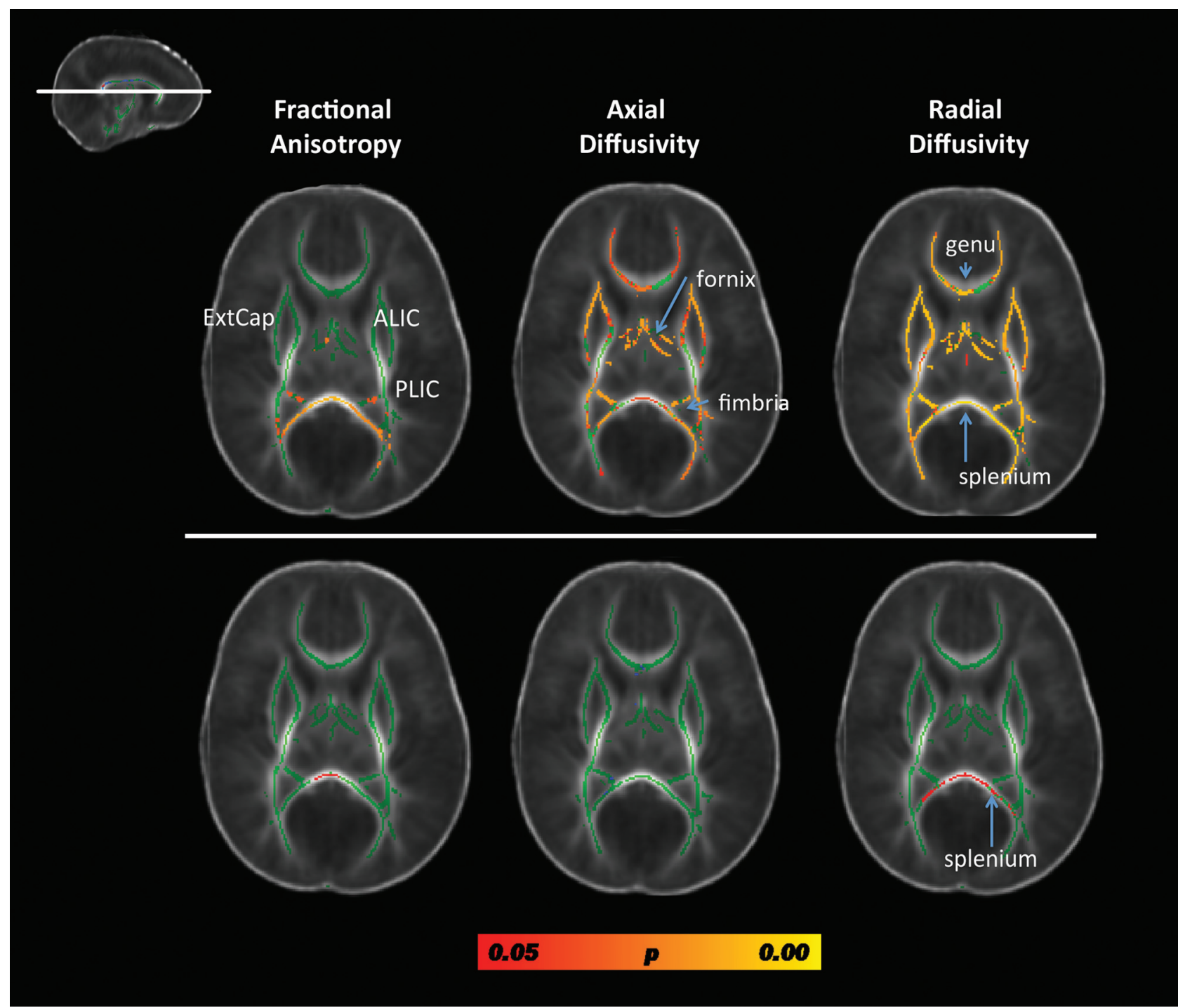

FIG 2. Results from the whole-brain, voxelwise TBSS analysis contrasting fractional anisotropy, axial diffusivity, and radial diffusivity metrics between preterm patients with CHD and term neonates without CHD, controlling for postconceptional age. Results from a single cut at the level of the genu and splenium of the corpus callosum are displayed (see inlay in the upper left). The top row includes data from comparing all preterm CHD cases to the term neonates without CHD. Diffuse microstructural abnormalities are seen in nearly all white matter regions. The bottom row includes data from comparing only the preterm CHD cases without PWMLs with the term neonates without CHD. The only structure showing micotructural abnormality is the splenium. Voxels showing a significant reduction in FA and axial diffusivity and a significant increase in radial diffusivity are shown in red-yellow, with the color bar denoting statistical significance, corrected for multiple comparisons. Note that most of the group differences are due to the cases with pWMLs or periventricular leukomalacia. ExtCap indicates external capsule; ALIC = anterior limb of the internal capsule; PLIC = posterior limb of the internal capsule.

\section{DISCUSSION}

Microstructural abnormalities and white matter injury, namely pWMLs, a putative MR imaging biomarker for noncavitary periventricular leukomalacia, have been well documented in previous studies of term neonates with CHD. ${ }^{9-12,16-19}$ This is the first study, to our knowledge, to use MR imaging and DTI to describe the occurrence of pWMLs and associated brain microstructural abnormalities in preterm neonates with CHD. Our most significant finding is that preterm neonates with CHD demonstrate diffuse microstructural changes in central white matter tracts (subserving visual, motor, limbic, and frontal executive functions) when compared with other critically ill neonates without CHD. However, these microstructural abnormalities were strongly associated with the presence of pWMLs. Importantly, we did not observe many diffuse microstructural differences between preterm infants with CHD and preterm infants without CHD when the infants with pWMLs were excluded. Moreover, after correction for number of pWMLs, there was no difference in microstructure of complex CHD cases compared with other heart lesions. These findings support the hypothesis that there are diffuse microstructural abnormalities in preterm CHD cases and indicate that the microstructural abnormalities are most strongly associated with white matter injury. Of note, independent of white matter injury, we identified a microstructural abnormality in the splenium of the corpus callosum in all preterm infants with CHD. The splenium is composed of white matter pathways connecting visual regions, and these microstructural differences may be a biomarker for vulnerability for visual spatial dysfunction, which 


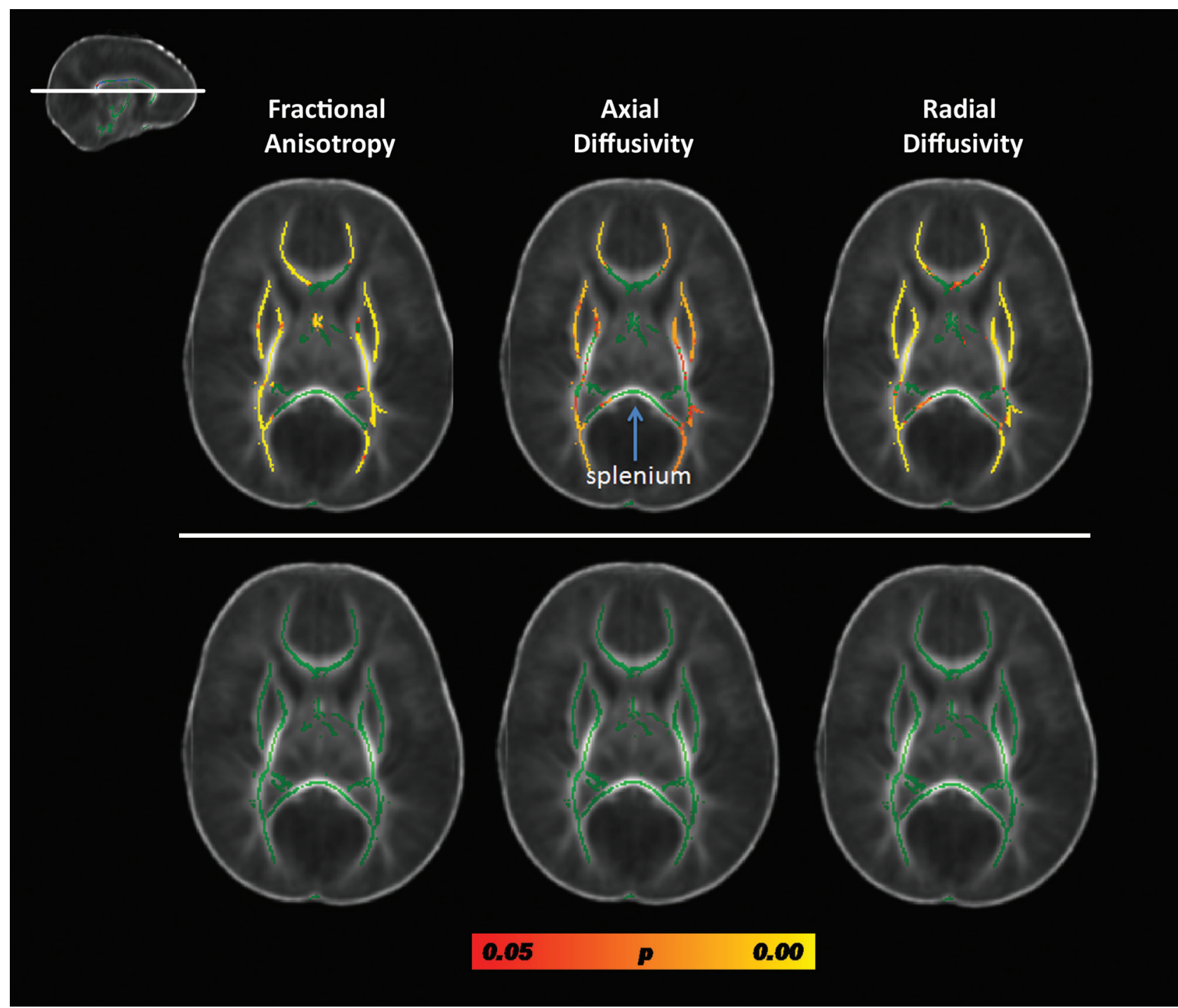

FIG 3. Results from the TBSS analysis contrasting FA, axial diffusivity (AD), and radial diffusivity (RD) in preterm patients with CHD with complex CHD lesions compared with preterm patients with CHD other heart lesions. Results from a single cut at the level of the genu and splenium of the corpus callosum are displayed (see inlay). The top row depicts the results of the contrasts between the preterm neonates with complex heart lesions relative to the preterm neonates with other heart lesions; the bottom row depicts the results from the contrasts of the same cases after correction for number of pWMLs. Both analyses are corrected for postconceptional age. Voxels showing a significant reduction in FA and AD and a significant increase in RD are shown in red-yellow, with the color bar denoting statistical significance, corrected for multiple comparisons. Of note, there is no difference in DTI metrics values in the splenium of the corpus callosum (arrow) between heart types.

is a well-described neurocognitive deficit observed in survivors of CHD. $^{27-29}$

Similar to prior studies of term infants with $\mathrm{CHD},{ }^{12}$ we observed diffuse microstructural abnormalities in our preterm infants with CHD compared with term infants without CHD. However, in our study, these differences were largely attributable to the presence of pWMLs rather than maturational lag as had been suggested by Miller et $\mathrm{al}^{12}$ in their analysis of term neonates with CHD. We did observe regional maturational differences (centered in the splenium); however, our widespread abnormalities in microstructure were only observed in the preterm neonates with CHD with pWMLs. This suggests that although the maturational state of the brain probably plays a role in the vulnerability to white matter injury, injury during the perinatal period and the associated microstructural abnormalities may best account for adverse neurodevelopmental outcomes in the preterm neonates with CHD. $^{30}$

A maturational delay in fetal brain development has been described in term neonates with complex CHD. ${ }^{13,31}$ Importantly, the available data suggest that the maturational delay accelerates during the late third trimester. ${ }^{13}$ Thus, for a cohort of neonates with CHD born on average at 32 weeks gestational age, it would not necessarily be expected that we would observe global maturational delays on the microstructure of the cerebral white matter arising from delayed intrauterine development. Other techniques including structural imaging techniques may be more sensitive to detect maturational differences in this population, as demonstrated previously in term CHD cases. ${ }^{14}$

Consistent with prior studies of $\mathrm{CHD}$, the highest incidence of pWMLs and concurrent microstructural abnormalities was 
found in the infants with hypoplastic left heart syndrome. It is well established that these infants are at exceedingly high risk for maturational delay and perinatal brain injury owing to the complex factors associated with abnormal uterine brain development and abnormal cerebral perfusion. ${ }^{10,11,32}$ However, not all of the infants with hypoplastic left heart syndrome have significant longterm neurocognitive problems, and initial results from the multicenter single ventricle reconstruction trial indicate that early neurodevelopmental outcome is more highly associated with innate patient variables than with intraoperative management strategies. ${ }^{33}$ In this analysis, there were no differences in microstructure between the neonates with complex CHD and neonates with other congenital heart lesions after correcting for the number of pWMLs. It is possible that pWMLs or associated microstructural abnormalities at the neonatal time period will be able to further distinguish among the infants with hypoplastic left heart syndrome, who are at highest risk for specific adverse neurodevelopmental outcomes.

Longitudinal studies of neonates with CHD have documented neurocognitive impairments across multiple cognitive domains, but most prominently in visual-spatial functions, executive functions, and language. ${ }^{27-29,34-38}$ In this study, widespread microstructural abnormalities involving neural systems subserving the visual system, motor system, limbic system, and frontoparietal executive system were identified in the preterm neonates with CHD with $\mathrm{pWMLs}$. These results suggest that irrespective of heart lesion, this subpopulation of preterm neonates with CHD would be most at risk for long-term neurocognitive deficits involving visual functions, motor development, emotions/behavioral regulation, and attention/executive functions. ${ }^{38}$

In contrast, the preterm neonates with $\mathrm{CHD}$ but without pWMLs were found to have microstructural abnormalities in the vicinity of the splenium - the posterior bundle of callosal crossing fibers. The splenium is one of the most rapidly developing white matter structures during the late fetal and early postnatal period. ${ }^{39}$ The splenium contains fiber bundles crossing between temporal and occipital regions of the left and right hemispheres that are important for normal visual-spatial functions. Accordingly, the finding of a microstructural abnormality in the preterm CHD cases without PVL compared with term controls suggests that the infants with CHD may be at increased risk for visualspatial dysfunction regardless of the presence of brain injury. ${ }^{27-29}$

We found that younger age at surgery and increased use of inotropes were associated with pWMLs in the preterm CHD group, similar to previous studies of pWMLs in term patients with CHD, suggesting a similar pathogenesis or vulnerability to white matter injury in both the preterm and term CHD groups. ${ }^{30}$ One remarkable clinical distinction between the preterm CHD group and both control groups was the high incidence of being small for gestational age in the preterm CHD group, suggesting that there could still be a prenatal influence on white matter vulnerability and injury in this group. ${ }^{40}$

\section{Limitations}

It could be suggested that we should have focused on the major left-sided heart lesions and excluded infants with other lesions such as atrial septal defect or ventricular septal defect due to marked differences in the hemodynamics in these neonates both in utero and postnatally. ${ }^{32}$ However, the incidence of hypoplastic left heart syndrome among preterm infants is relatively rare and we did not have sufficient sample size to include these lesions alone. A second limitation in this study is the inclusion of cases with MR imaging scans performed preoperatively and postoperatively. Third, there is the potential for sampling bias as we relied on clinical scans to identify our neonates before induction. Fourth, our study sample size was small and recruited from a single institution.

\section{CONCLUSIONS}

Our study provides evidence that preterm neonates with $\mathrm{CHD}$ demonstrate diffuse microstructural changes in central white matter tracts when compared with other critically ill neonates without CHD. However, these microstructural changes are highly associated with the occurrence of pWMLs, a biomarker for noncavitary periventricular leukomalacia. Regardless of injury, a persistent microstructural abnormality was noted in the splenium, which could explain the high prevalence of visual-spatial neurocognitive dysfunction in surviving patients with CHD. This study suggests that MR imaging may not only have a beneficial role in identifying preterm infants with $\mathrm{CHD}$ who are at the greatest risk for adverse neurodevelopmental outcome, but also may be a clinical biomarker to help guide potential neuroprotective or rehabilitative therapies in this population.

\section{ACKNOWLEDGMENTS}

The authors thank Julie Castro, Anita Hamilton, and Hesham Mahmoud for their assistance with patient recruitment and data management. We also thank Drs Hannah Kinney and Dr Istvan Seri for careful review of the manuscript. We thank Melanie Gieraltowski for manuscript preparation.

Disclosures: RELATED: Grant: NINDS (listed under grant funding)* $\left({ }^{*}\right.$ money paid to institution)

\section{REFERENCES}

1. Marino BS, Lipkin PH, Newburger JW, et al. Neurodevelopmental outcomes in children with congenital heart disease: evaluation and management: a scientific statement from the American Heart Association. Circulation 2012;126:1143-72

2. Tanner K, Sabrine N, Wren C. Cardiovascular malformations among preterm infants. Pediatrics 2005;116:e833-e838

3. Dees E, Lin H, Cotton RB, et al. Outcome of preterm infants with congenital heart disease. J Pediatr 2000;137:653-59

4. Cheng $\mathrm{HH}$, Almodovar MC, Laussen PC, et al. Outcomes and risk factors for mortality in premature neonates with critical congenital heart disease. Pediatr Cardiol 2011;32:1139-46

5. Natarajan G, Anne SR, Aggarwal S. Outcomes of congenital heart disease in late preterm infants: double jeopardy? Acta Paediatr 2011;100:1104-07

6. Gelehrter S, Fifer CG, Armstrong A, et al. Outcomes of hypoplastic left heart syndrome in low-birth-weight patients. Pediatr Cardiol 2011;32:1175-81

7. Costello JM, Polito A, Brown DW, et al. Birth before 39 weeks' gestation is associated with worse outcomes in neonates with heart disease. Pediatrics 2010;126:277-84

8. Goff DA, Luan X, Gerdes M, et al. Younger gestational age is associated with worse neurodevelopmental outcomes after cardiac surgery. J Thorac Cardiovasc Surg 2012;143:535-42 
9. Andropoulos DB, Hunter JV, Nelson DV, et al. Brain immaturity is associated with brain injury before and after neonatal cardiac surgery with high-flow bypass and cerebral oxygenation monitoring. J Thorac Cardiovasc Surg 2010;139:543-56

10. McQuillen PS, Miller SP. Congenital heart disease and brain development. Ann N Y Acad Sci 2010;1184:68-86

11. McQuillen PS, Goff DA, Licht DJ. Effects of congenital heart disease on brain development. Prog Pediatr Cardiol 2010;29:79-85

12. Miller SP, McQuillen PS, Hamrick S, et al. Abnormal brain development in newborns with congenital heart disease. $N$ Engl J Med 2007;357:1928-38

13. Limperopoulos C, Tworetzky W, McElhinney DB, et al. Brain volume and metabolism in fetuses with congenital heart disease: evaluation with quantitative magnetic resonance imaging and spectroscopy. Circulation 2010;121:26-33

14. Licht DJ, Shera DM, Clancy RR, et al. Brain maturation is delayed in infants with complex congenital heart defects. $J$ Thorac Cardiovasc Surg 2009;137:529-37

15. Watanabe K, Matsui M, Matsuzawa J, et al. Impaired neuroanatomic development in infants with congenital heart disease. J Thorac Cardiovasc Surg 2009;137:146-53

16. Galli KK, Zimmerman RA, Jarvik GP, et al. Periventircular leukomalacia is common after neonatal cardiac surgery. $J$ Thorac Cardiovasc Surg 2004;127:692-704

17. Mahle WT, Tavani F, Zimmerman RA, et al. An MRI study of neurological injury before and after congenital heart surgery. Circulation 2002;106:I109-I114

18. Partridge SC, Vigneron DB, Charlton NN, et al. Pyramidal tract maturation after brain injury in newborns with heart disease. Ann Neurol 2006;59:640-51

19. Kinney HC, Panigrahy A, Newburger JW, et al. Hypoxic-ischemic brain injury in infants with congenital heart disease dying after surgery. Acta Neuropathol 2005;110:563-78

20. Miller SP, Ferriero DM, Leonard C, et al. Early brain injury in premature newborns detected with magnetic resonance imaging is associated with adverse neurodevelopmental outcomes. J Pediatr 2005;147:609-16

21. Nanba Y, Matsui K, Aida N, et al. Magnetic resonance imaging regional $\mathrm{T} 1$ abnormalities at term accurately predicts motor outcome in preterm infants. Pediatrics 2007;120:e10-e19

22. Buser JR, Maire J, Riddle A, et al. Arrested preoligodendrocyte maturation contributes to myelination failure in premature infants. Ann Neurol 2012;71:93-109

23. Miller SP, Ferriero DM. From selective vulnerability to connectivity: insight from newborn brain imaging. Trends Neurosci 2009;32:496-505

24. Bartha AI, Yap KR, Miller SP, et al. The normal neonatal brain: MR imaging, diffusion tensor imaging, and 3D MR spectroscopy in healthy term neonates. AJNR Am J Neuroradiol 2007;28:1015-21
25. Smith SM. Tract-based spatial statistics: voxelwise analysis of multi-subject diffusion data. Neuroimage 2006;31:1487-505

26. Ball G, Counsell SJ, Anjari M, et al. An optimised tract-based spatial statistics protocol for neonates: applications to prematurity and chronic lung disease. Neuroimage 2010;53:94-102

27. Bellinger DC, Newburger JW. Neuropsychological, psychosocial, and quality-of-life outcomes in children and adolescents with congenital heart disease. Prog Pediatr Cardiol 2010;29:87-92

28. Kravitz DJ, Saleem KS, Baker CI, et al. A new neural framework for visuospatial processing. Nat Rev Neurosci 2011;12:217-30

29. Bellinger DC, Bernstein JH, Kirkwood MW, et al. Visual-spatial skills in children after open-heart surgery. J Dev Behav Pediatr 2003;24:169-79

30. Ishibashi N, Scafidi J, Murata A, et al. White matter protection in congenital heart surgery. Circulation 2012;125:859-71

31. Clouchoux C, du Plessis AJ, Bouyssi-Kobar M, et al. Delayed cortical development in fetuses with complex congenital heart disease. Cereb Cortex 2012 Sep 12. [Epub ahead of print]

32. Kaltman JR, Di H, Tian Z, et al. Impact of congenital heart disease on cerebrovascular blood flow dynamics in the fetus. Ultrasound Obstet Gynecol 2005;25:32-36

33. Newburger JW, Sleeper LA, Bellinger DC, et al. Early developmental outcome in children with hypoplastic left heart syndrome and related anomalies: the single ventricle reconstruction trial. Circulation 2012;125:2081-91

34. Ballweg JA, Wernovsky G, Gaynor JW. Neurodevelopmental outcomes following congenital heart surgery. Pediatr Cardiol 2007; 28:126-33

35. Bellinger DC, Wypij D, Rivkin MJ, et al. Adolescents with d-transposition of the great arteries corrected with the arterial switch procedure: neuropsychological assessment and structural brain imaging. Circulation 2011;124:1361-69

36. Mahle WT, Clancy RR, Moss EM, et al. Neurodevelopmental outcome and lifestyle assessment in school-aged and adolescent children with hypoplastic left heart syndrome. Pediatrics 2000; 105:1082-89

37. Karsdorp PA, Everaerd W, Kindt M, et al. Physiological and cognitive functioning in children and adolescents with congenial heart disease. Pediatr Psychol 2007;32:527-41

38. Shillingford AJ, Glanzman MM, Ittenbach RF, et al. Inattention, hyperactivity, and school performance in a population of school-age children with complex congenital heart disease. Pediatrics 2008; 121:e759-e767

39. Kasprian G, Brugger PC, Weber M, et al. In utero tractography of fetal white matter development. Neuroimage 2008;43:213-24

40. Padilla N, Falcón C, Sanz-Cortés M, et al. Differential effects of intrauterine growth restriction on brain structure and development in preterm infants: a magnetic resonance imaging study. Brain Res 2011;1382:98-108 